\title{
Performance of an Autonomous Energy Harvesting Wireless Sensor
}

\author{
Erol Gelenbe, FIEEE, and Yasin M. Kadioglu \\ Intelligent Systems and Networks Group \\ Dept. of Electrical and Electronic Engineering \\ Imperial College, London SW7 2BT, UK \\ \{e.gelenbe,y.kadioglu14\}@imperial.ac.uk
}

\begin{abstract}
While complex autonomic self-organising systems have received much attention, simple autonomous systems are also needed for remote sensing applications, as well as for the the Internet of Things. Such autonomous stand-alone unattended devices may not have access to reliable sources of mains power, and will have to harvest energy locally from ambient sources such as vibrations, heat or light. However energy leakage will also be a problem. This paper proposes a mathematical model to analyse the performance of such systems in the presence of a random source of energy, as well as a random source of data. The equilibrium between random energy, random data and random leakage results in an interesting performance analysis of these small but ubiquitous systems as a whole. A discussion is also provided about the effect of transmission errors.
\end{abstract}

Index Terms-Autonomous Energy Harvesting; Performance Evaluation; Autonomous Wireless Sensor

\section{INTRODUCTION AND PREVIOUS WORK}

In recent years, much work has been conducted on selforganised or "autonomic" communication systems [1] and biologically inspired [2] or adaptive control methods [3] have been suggested for their management. However many standalone autonomous systems require a very simple organisation for unattended long term operation. One such area of application is in stand-alone wireless sensors which need to operate remotely without a change of batteries. Such systems are also motivated by the cost or difficulty of access, and also by the potential environmental damage when one discards batteries, and by the need to save energy in ICT [4], [5].

Thus energy harvesting from solar, thermal, vibrationial, or ambient electromagnetic radiation including light, are of particular interest [6], [7], especially in remote sensing and security applications [8], [9], [10], and recent research has addressed such technologies for communications [11]. However much work still needs to be done to understand the performance of such systems which need to operate autonomously for very long periods of time.

Earlier work [12] studied the performance of an autonomous energy harvesting communication node as a function of the random flow of harvested energy using an "energy packet" model which discretises both the data flow and the energy flow in the sensor node [13] based on queueing networks [14]. Here we extend the work to study the ability of such a system to operate in an unattended and autonomous manner, in the presence of leakage from energy storage units such as batteries or capacitors.

\section{A. The mathematical model}

An energy harvesting wireless sensor communication node collects data to transmit atrate $\lambda$ data packets per second from sensing activities, and harvests energy at rate $\Lambda$ energy packets per second, where one energy packet is the unit of electrical energy, e.g. micro-joules, collected from light, heat or vibrations, that is needed to transmit one data packet. The energy harvesting rate $\Lambda$ and data gathering rate $\lambda$ are assumed to be small (i.e. very slow) as compared to the time it takes to create and transmit a packet via wireless, which may be in the nano or picoseconds. The node stores energy in a capacitor or battery, and energy leaks in a random manner at rate of $\mu$ energy packets per second.

The state of the system is represented by $K(t)$, the number of data packets stored at the node, and by $M(t)$ the number of energy packets that are stored, at at time $t \geq 0$. Since the transmission time at the node is very short, whenever energy is available and there are data packets waiting they will be instantaneously transmitted till the energy is depleted: from a state $K(t)>0, M(t)>0$ the system instantaneously moves to either state $(0, M(t)-K(t))$ if $M(t) \geq K(t)$, or to $(K(t)-$ $M(t), 0)$ if $K(t) \geq M(t)$. Writing $p(n, m, t)=\operatorname{Prob}[K(t)=$ $n, M(t)=m]$, we therefore only consider $p(n, m, t)$ for pairs of integers $(n, m) \in S$ with

$$
S=\{(0,0),(n, 0),(0, m): n>0, m>0\} .
$$

\section{Finite CAPACity DATA AND ENERgy BufFers With ENERGY LEAKAGE}

First note that if both the data and energy If both the data buffer and the energy storage capacity are finite, the system can be modelled as a finite Markov chain whose set of states are given in (1) with $0 \leq n \leq B, 0 \leq m \leq E$. We note that the process $[K(t), M(t), t \geq 0]$ is an irreducible irreducible and aperiodic Markov chain so that the stationary probabilities $p(n, m)=\lim _{t \rightarrow \infty} \operatorname{Pr}[K(t)=n, M(t)=m]$ exist and are computed from the equations:

$$
p(0,0)[\lambda+\Lambda]=\Lambda p(1,0)+\lambda p(0,1)+\mu p(0,1),
$$

since state $(0,0)$ is reached if either there was just one data packet and it was transmitted as soon as an energy packet 
arrived, or there was one energy packet and it was consumed as soon as a data packet arrived, or one energy packet was lost due to leakage. When $0<n<B$ we have:

$$
p(n, 0)[\lambda+\Lambda]=\Lambda p(n+1,0)+\lambda p(n-1,0),
$$

while:

$$
p(B, 0) \Lambda=p(B-1,0) \lambda
$$

We note that these equations have a solution of the form:

$$
p(n, 0)=\alpha^{n} C_{d}, \alpha=\frac{\lambda}{\Lambda},
$$

where $C_{d}$ is a constant. For the energy storage system, when $0<m<E$ we have:

$p(0, m)[\lambda+\Lambda+\mu]=\Lambda p(0, m-1)+\lambda p(0, m+1)+\mu p(0, m+1)$

while:

$$
p(0, E)[\lambda+\mu]=p(0, E-1) \Lambda .
$$

We note that these equations have a solution of the form:

$$
p(0, m)=\theta^{m} C_{e}, \theta=\frac{\Lambda}{\lambda+\mu},
$$

where $C_{e}$ is a constant. Since the unique stationary probability distribution exists, we must have $C_{d}=C_{e}=p(0,0)$, and considering equation (2) we obtain:

$$
\begin{array}{r}
p(0,0)(\lambda+\Lambda)=\Lambda\left(\frac{\lambda}{\Lambda}\right) C_{d}+(\lambda+\mu)\left(\frac{\Lambda}{\lambda+\mu}\right) C_{e} \\
0=\left(p(0,0)-C_{d}\right) \lambda+\left(p(0,0)-C_{e}\right) \Lambda .
\end{array}
$$

Using the fact that the probabilities sum to one we have:

$$
\begin{aligned}
1 & =p(0,0)+\sum_{n=1}^{B} p(n, 0)+\sum_{m=1}^{E} p(0, m), \\
& =p(0,0)\left[1+\sum_{n=1}^{B} \alpha^{n}+\sum_{m=1}^{E} \theta^{m}\right], \\
& =p(0,0)\left[1+\left(\frac{\alpha\left(\alpha^{B}-1\right)}{\alpha-1}\right)+\left(\frac{\theta\left(\theta^{E}-1\right)}{\theta-1}\right) .\right.
\end{aligned}
$$

Hence:

$$
\begin{array}{r}
p(0,0)=\frac{1-\alpha-\theta+\alpha \theta}{\alpha^{B+1}(\theta-1)+\theta^{E+1}(\alpha-1)+1-\alpha \theta}, \\
p(n, 0)=\alpha^{n} \frac{1-\alpha-\theta+\alpha \theta}{\alpha^{B+1}(\theta-1)+\theta^{E+1}(\alpha-1)+1-\alpha \theta}, \\
p(0, m)=\theta^{m} \frac{1-\alpha-\theta+\alpha \theta \quad 0 \leq n \leq B,}{\alpha^{B+1}(\theta-1)+\theta^{E+1}(\alpha-1)+1-\alpha \theta}, \\
0 \leq m \leq E .
\end{array}
$$

Also, we can express the marginal probabilities for the queue length of DPs and EPs as:

$$
\begin{array}{r}
p_{d}(n)= \\
\sum_{m=0}^{\infty} p(n, m)=p(n, 0), n>0, \\
p_{d}(0)= \\
\sum_{m=0}^{\infty} p(0, m)=\sum_{m=0}^{\infty} \theta^{m} p(0,0)=\frac{1-\theta^{E+1}}{1-\theta} p(0,0),
\end{array}
$$

and similarly,

$$
\begin{array}{r}
p_{e}(m)= \\
\sum_{n=0}^{\infty} p(n, m)=p(0, m), m>0 \\
p_{e}(0)= \\
\sum_{n=0}^{\infty} p(n, 0)=\sum_{n=0}^{\infty} \alpha^{n} p(0,0)=\frac{1-\alpha^{B+1}}{1-\alpha} p(0,0) .
\end{array}
$$

Hence:

$$
\begin{array}{r}
p_{d}(n)=\alpha^{n} \frac{1-\alpha-\theta+\alpha \theta}{\alpha^{B+1}(\theta-1)+\theta^{E+1}(\alpha-1)+1-\alpha \theta}, \\
0<n \leq B, \\
p_{e}(m)=\theta^{m} \frac{1-\alpha-\theta+\alpha \theta}{\alpha^{B+1}(\theta-1)+\theta^{E+1}(\alpha-1)+1-\alpha \theta}, \\
0<m \leq E .
\end{array}
$$

\section{EnERgy And DAta PACKet Loss due to Finite ENERGY AND DATA BUFFERS}

When the energy storage capacity is finite, or the data packet buffer is finite, we are bound to have some level of energy loss or data packet loss. These loss rates $L_{e}, L_{d}$ in energy and data packets per second, respectively, can easily be computed as:

$$
\begin{aligned}
L_{e} & =\Lambda \sum_{n=0}^{\infty} p(n, E)=\Lambda p(0, E), \\
& =\Lambda \theta^{E} \frac{1-\alpha-\theta+\alpha \theta}{\alpha^{B+1}(\theta-1)+\theta^{E+1}(\alpha-1)+1-\alpha \theta} . \\
L_{d} & =\lambda \sum_{m=0}^{\infty} p(B, m)=\lambda p(B, 0), \\
& =\lambda \alpha^{B} \frac{1-\alpha-\theta+\alpha \theta}{\alpha^{B+1}(\theta-1)+\theta^{E+1}(\alpha-1)+1-\alpha \theta} .
\end{aligned}
$$

so that after some algebra we get:

$$
\begin{aligned}
L_{e} & =\frac{\Lambda-\lambda}{1-\alpha^{B+1} \theta^{-E}}, \\
L_{d} & =\frac{\lambda(1-\theta)}{1-\theta^{E+1} \alpha^{-B}} .
\end{aligned}
$$

For the assumption of very large buffer sizes i.e., both B and E tend to infinity, the following cases can be considered:

Case 1 If $\alpha>1$ and hence $\theta<1$ or equivalently $\Lambda-\mu<\lambda$, so that the energy is not sufficient enough for the data and $L_{e} \rightarrow 0$ and $L_{d} \rightarrow \lambda(1-\theta)$, as would be expected. 
Case 2 If $\alpha=1$ and hence $\theta<1$ or equivalently $\Lambda-\mu<\lambda$, similar from the Case $1, L_{e} \rightarrow 0$ and $L_{d} \rightarrow \lambda(1-\theta)$.

Case 3 If $\alpha<1$ and $\theta>1$ or equivalently $\Lambda>\lambda+\mu$, so that the energy is more plentiful than it is needed, and $L_{e} \rightarrow \Lambda-\lambda$ and $L_{d} \rightarrow 0$, as would be expected.

Case 4 If $\alpha<1$ and $\theta=1$ or equivalently $\Lambda-\lambda=\mu$, so that we can see the difference between arrival rate of the energy packets and the effective arrival rate of the data packets is exactly related with the leakage rate, and $L_{e} \rightarrow \mu$ and $L_{d} \rightarrow 0$, as would be expected.

\section{A. Stability}

As in [12], system stability is only of interest for data and energy buffers with unlimited storage capacity. In this case, the system will be stable when the backlog of stored energy and the backlog of data packets remain finite with probability one when $t \rightarrow \infty$ for $B \rightarrow \infty$ and $E \rightarrow \infty$. Otherwise the system will be said to be unstable.

Now for some finite $G$ and $H$ with $0 \leq G<B$ and $0 \leq$ $H<E$, the probabilities that the respective backlogs of data and energy packets do not exceed $G$ and $H$, respectively, in steady-state:

$$
\begin{aligned}
& P_{d}(G)=\lim _{t \rightarrow \infty} \operatorname{Prob}[0 \leq K(t) \leq G \leq B] \\
& P_{e}(H)=\lim _{t \rightarrow \infty} \operatorname{Prob}[0 \leq M(t) \leq H \leq E]
\end{aligned}
$$

so that using our previous results we have:

$$
\begin{aligned}
P_{d}(G) & =p_{d}(0)+\sum_{n=1}^{G} p_{d}(n), \\
& =\frac{1-\theta^{E+1}}{1-\theta} p(0,0)+\sum_{n=1}^{G}\left(\alpha^{n} p(0,0)\right), \\
& =p(0,0)\left(\frac{1-\theta^{E+1}}{1-\theta}+\frac{\alpha-\alpha^{G+1}}{1-\alpha}\right), \\
& =\frac{\alpha^{G+1}(\theta-1)+\theta^{E+1}(\alpha-1)+1-\alpha \theta}{\alpha^{B+1}(\theta-1)+\theta^{E+1}(\alpha-1)+1-\alpha \theta}
\end{aligned}
$$

and

$$
\begin{aligned}
P_{e}(H) & =p_{e}(0)+\sum_{m=1}^{G} p_{e}(m) \\
& =\frac{1-\alpha^{B+1}}{1-\alpha} p(0,0)+\sum_{m=1}^{H}\left(\theta^{m} p(0,0)\right) \\
& =p(0,0)\left(\frac{1-\alpha^{B+1}}{1-\alpha}+\frac{\theta-\theta^{H+1}}{1-\theta}\right) \\
& =\frac{\alpha^{B+1}(\theta-1)+\theta^{H+1}(\alpha-1)+1-\alpha \theta}{\alpha^{B+1}(\theta-1)+\theta^{E+1}(\alpha-1)+1-\alpha \theta}
\end{aligned}
$$

This leads to the following:

Case 1 If $\alpha>1$ and hence $\theta<1$ as $E \rightarrow \infty$ and $B \rightarrow \infty$, $P_{d}(G) \rightarrow 0$ for all finite $G$ and $P_{e}(H) \rightarrow 1$ for all finite $H$, the system is stable with respect to EPs and unstable with respect to DPs.
Case 2 If $\alpha=1$ and hence $\theta<1$ as $E \rightarrow \infty$ and $B \rightarrow \infty$, one can easily see that the expression for $p(0,0)$ is an indeterminate form, so that we apply L'Hospital's rule and obtain :

$$
\begin{array}{r}
\lim _{\alpha \rightarrow 1} p(0,0)= \\
\lim _{\alpha \rightarrow 1} \frac{\theta-1}{(B+1)(\theta-1) \alpha^{B}+\theta^{E+1}-\theta}= \\
\frac{\theta-1}{(B+1)(\theta-1)+\theta^{E+1}-\theta}, \\
\lim _{(B, E) \rightarrow \infty}\left[\lim _{\alpha \rightarrow 1} p(0,0)\right] \rightarrow 0 .
\end{array}
$$

Hence:

$$
\begin{array}{r}
\lim _{(B, E) \rightarrow \infty, \alpha \rightarrow 1}\left[P_{d}(G)\right]= \\
\lim _{(B, E) \rightarrow \infty, \alpha \rightarrow 1}\left[\sum_{m=0}^{E}\left(\theta^{m} p(0,0)\right)+\sum_{n=1}^{G}\left(\alpha^{n} p(0,0)\right)\right]= \\
\lim _{(B, E) \rightarrow \infty, \alpha \rightarrow 1}[p(0,0)] \lim _{(B, E) \rightarrow \infty, \alpha \rightarrow 1}\left[\sum_{m=0}^{E} \theta^{m}+\sum_{n=1}^{G} \alpha^{n}\right], \\
\lim _{(B, E) \rightarrow \infty, \alpha \rightarrow 1} P_{d}(G) \rightarrow 0 .
\end{array}
$$

in this case a similar analysis can be made for the $P_{e}(H)$ and which leads $P_{d}(G) \rightarrow 0$ for all finite $G$ and $P_{e}(H) \rightarrow 0$ for all finite $H$. Therefore, the system is unstable with respect to both DPs and EPs.

Case 3 If $\alpha<1$ and $\theta<1$ as $E \rightarrow \infty$ and $B \rightarrow \infty$, $P_{d}(G) \rightarrow \frac{\alpha^{G+1}(\theta-1)+1-\alpha \theta}{1-\alpha \theta}$ and that limit is obviously in the interval $(0,1)$, since $-1<\alpha^{G+1}(\theta-1)<0$ for all finite $G$ and similarly $P_{e}(H) \rightarrow \frac{\theta^{H+1}(\alpha-1)+1-\alpha \theta}{1-\alpha \theta}$ and similarly that limit is in the interval $(0,1)$, for all finite $H$. Therefore, the system is unstable with respect to both DPs and EPs.

Case 4 If $\alpha<1$ and hence $\theta=1$ as $E \rightarrow \infty$ and $B \rightarrow \infty$, one can easily see that the expression for $p(0,0)$ is an indeterminate form, so that the analysis in this case can be made by applying exact same procedure which is done in Case 2. Thewrefore, since $P_{d}(G) \rightarrow 0$ for all finite $G$ and $P_{e}(H) \rightarrow 0$ for all finite $H$, the system is unstable with respect to both DPs and EPS.

Case 5 If $\alpha<1$ and $\theta>1$ as $E \rightarrow \infty$ and $B \rightarrow \infty$, $P_{d}(G) \rightarrow 1$ for all finite $G$ and $P_{e}(H) \rightarrow 0$ for all finite $H$; the system is stable with respect to DPs and unstable with respect to EPs.

\section{SEnsors With TRANSMISSION ERRORS}

When a transmission error is detected, retransmission of the same data packet may be needed because a transmission error has been detected, and is modelled as follows. If a data packet is waiting in queue and an energy packet arrives, so that the data packet can be transmitted, then a transmission error occurs with probability $\pi$ and the data packet is not deleted from the queue. Similarly, if a data packet arrives to the node when one or more energy packets are waiting, then a transmission error may occur with probability $p$ and the transmission will be repeated independently with the 
probability of correct transmission $(1-p)$ and error $p$, and this will be repeated until success occurs or until all the energy packets are depleted. As a result, the state transition rates are: - $\lambda:$ for $(n, 0) \rightarrow(n+1,0), n \geq 0$, when a data packet arrives,

- $\Lambda:$ for $(0, m) \rightarrow(0, m+1), m \geq 0$, when an energy packet arrives,

- $\mu:$ for $(0, m) \rightarrow(0, m-1), m \geq 1$, when an energy packet leaves due to leakage.

- $\Lambda \pi:$ for $(n, 0) \rightarrow(n, 0), n \geq 1$, when an energy packet arrives to an empty energy buffer, we have this transition, because upon arrival of an energy packet to an empty energy buffer, if the data packet transmission is immediately followed by the request for another data packet transmission with probability $\pi$, then the new data packet will not be transmitted due to lack of an energy packet and will simply replace the previous one.

$-\Lambda(1-\pi):$ for $(n, 0) \rightarrow(n-1,0), n \geq 1$, if the data packet transmission (caused by the arrival of an energy packet) is not followed by another data packet transmission request, which occurs with probability $(1-\pi)$, then we have this transition. - $\lambda p:$ for $(0,1) \rightarrow(1,0)$, when a data packet arrives, because the energy packet in queue serves the transmission of the arriving energy data packet, an additional data packet transmission is needed with probability $p$, and the data packet will have to wait for another energy packet to arrive.

Finally there will be a set of transitions starting from states of the form $(0, m), m>0$, when the arrival of a data packet results in a sequence of $k$ successive repetitions of data packets arriving to the node with probability $p^{k}(1-p)$ where $m \geq k \geq 0$, namely:

- $\lambda(1-p):$ for $(0, m) \rightarrow(0, m-1), m>0$,

- $\lambda p^{k-1}(1-p):$ for $(0, m) \rightarrow(0, m-k), m \geq k>1$,

and finally in the last case below, the arriving data packet reduces the number of energy packets by 1 and generates one additional data packet transition which is also transmitted, then there may be another data packet transmission request and so on, but when all the energy packets are depleted, the $m-t h$ and final transmission request cannot be satisfied and the system moves into state $(1,0)$ having depleted all its energy packets and having one final data packet waiting to be transmitted:

- $\lambda p^{m}:$ for $(0, m) \rightarrow(1,0)$, i.e. $k=m$, and notice that for any $m>0$ the sum of these probabilities is one:

$$
\sum_{k=0}^{m-1} p^{k}(1-p)+p^{m}=1
$$

These transitions lead to the equilibrium equations:

$$
\begin{array}{r}
p(0,0)[\lambda+\Lambda]= \\
\lambda \sum_{l=1}^{\infty} p^{l-1}(1-p) p(0, l)+\Lambda(1-\pi) p(1,0)+\mu p(0,1), \\
p(1,0)[\lambda+\Lambda(1-\pi)]= \\
\lambda \sum_{l=0}^{\infty} p^{l} p(0, l)+\Lambda(1-\pi) p(2,0), \\
p(n, 0)[\lambda+\Lambda(1-\pi)]= \\
\lambda p(n-1,0)+\Lambda(1-\pi) p(n+1,0), n>1, \\
\quad p(0, m)[\lambda+\Lambda+\mu]= \\
\lambda \sum_{l=1}^{\infty} p^{l-1}(1-p) p(0, m+l)+ \\
\Lambda p(0, m-1)+\mu p(0, m+1), m>0 .
\end{array}
$$

Theorem If $(\Lambda-\mu)(1-p)<\lambda<\Lambda(1-\pi)$, the stationary distribution exists and is given by:

$$
\begin{aligned}
p(0, m) & =p(0,0) Q^{m}, m \geq 0, \\
p(n, 0) & =p(1,0) q^{n-1}, n \geq 1,
\end{aligned}
$$

where

$$
Q=\frac{\lambda+\mu+\Lambda p-\sqrt{(\lambda+\mu+\Lambda p)^{2}-4 \mu \Lambda p}}{2 \mu p},
$$

and

$$
\begin{aligned}
& \frac{p(1,0)}{p(0,0)}=\frac{q}{(1-p Q)}= \\
& \frac{\lambda 2 \mu}{\Lambda(1-\pi)\left[\mu-\lambda-\Lambda p+\sqrt{(\lambda+\Lambda p+\mu)^{2}-4 \mu \Lambda p}\right]},
\end{aligned}
$$

with

$$
\begin{array}{r}
p(0,0)=\frac{(1-q)(1-Q)(1-p Q)}{q(1-Q)+(1-q)(1-p Q)}= \\
\left(\frac{\frac{2 \mu \lambda}{(\Lambda(1-\pi)-\lambda)}}{\left[\mu-\lambda-\Lambda p+\sqrt{(\lambda+\Lambda p+\mu)^{2}-4 \mu \Lambda p}\right]}+\right. \\
\left.\frac{2 \mu p}{2 \mu p-(\lambda+\Lambda p+\mu)+\sqrt{(\lambda+\mu+\Lambda p)^{2}-4 \mu \Lambda p}}\right)^{-1} .
\end{array}
$$

Proof To proceed with the proof, we substitute (48a) in (47), which after some algebra becomes:

$$
\begin{array}{r}
Q^{m}[\lambda+\Lambda+\mu]= \\
\lambda(1-p) Q^{m+1} \frac{1}{1-p Q}+\Lambda Q^{m-1}+\mu Q^{m+1}, \\
0=(Q-1)\left[Q^{2}(\mu p)+Q(-\Lambda p-\lambda-\mu)+\Lambda\right], \\
Q_{1,2}=\frac{\lambda+\mu+\Lambda p \pm \sqrt{(\lambda+\mu+\Lambda p)^{2}-4 \mu \Lambda p}}{2 \mu p},
\end{array}
$$


note that $Q$ has to be smaller than 1, while:

$$
\begin{aligned}
Q_{1} & =\frac{\lambda+\mu+\Lambda p+\sqrt{(\lambda+\mu+\Lambda p)^{2}-4 \mu \Lambda p}}{2 \mu p}, \\
& \geq \frac{\lambda+\mu+\Lambda p}{2 \mu p}, \\
& >\frac{1}{2}\left(\frac{1}{p}+\frac{\Lambda}{\mu}\right)>1,
\end{aligned}
$$

since $p<1$ and $\mu<\Lambda$, the root $Q_{1}$ should not be considered, and $Q_{2}$ is the only viable root. Now define $\varphi=(\lambda+\mu+\Lambda p)$ :

$$
\begin{aligned}
Q=\frac{\varphi-\sqrt{\varphi^{2}-4 \mu \Lambda p}}{2 \mu p} & <1, \\
\varphi^{2}-4 \varphi \mu p+4 \mu^{2} p^{2} & <\varphi^{2}-4 \mu \Lambda p, \\
\Lambda+\mu p & <\varphi, \\
(\Lambda-\mu)(1-p) & <\lambda .
\end{aligned}
$$

Also we must have $q<1$ and we note that the equation in (48b) has a solution of the form:

$$
\begin{array}{r}
p(n, 0)=q^{n-1} C_{d}, \\
q=\frac{\lambda}{\Lambda(1-\pi)} \text { and } C_{d}=p(1,0) \text { with } \lambda<\Lambda(1-\pi) .
\end{array}
$$

After further analysis using the fact that the probabilities must sum to one, and $q<1, Q<1$, we obtain:

$$
\begin{gathered}
\left(\frac{2 \mu \lambda}{(\Lambda(1-\pi)-\lambda)} \quad p(0,0)=\right. \\
\left.\frac{2 \mu p}{2 \mu p-(\lambda+\Lambda p+\mu)+\sqrt{(\lambda+\mu+\Lambda p)^{2}-4 \mu \Lambda p}}\right)^{-1} . \\
\text { V. ConCLUSIONS }
\end{gathered}
$$

Complex autonomic self-organising systems have been at the centre of attention over the last decade and have included several EU research projects and have met with wide interest in the literature. On the other hand, simple simple autonomous systems which have to operate in unattended remote environments have met with less research interest. Yet important areas such as remote sensing applications, as well as for the the Internet of Things, require that unattended autonomous systems operate reliably over long periods of time.

One important are of application is when one cannot change batteries of simple devices which may also be difficult to connect to the mains for their power needs. In such cases, the autonomous systems will have to harvest energy locally from ambient sources such as vibrations, heat or light, and energy leakage from temporary storage units, such as rechargeable batteries or capacitors, will also be a problem.

This paper analyses the performance of such systems that are devoted to gathering data and transmitting it, and which use energy harvesting for their operation. We have proposed a mathematical model to analyse the performance of such systems in the presence of a random source of energy, a random source of data, and random energy leakage. The equilibrium between random energy, random data and random leakage results in an interesting performance analysis of these small but ubiquitous systems. A preliminary discussion has also been given about how transmission errors can be included in the model.

Future work will have to incorporate the actual transmission scheme, including noise and interference, to better understand the optimum autonomous performance of such systems

\section{ACKNOWLEDGEMENTS}

The authors gratefully acknowledge the support of the ERA-NET ECROPS Project under grant No. Grant No. EP/K017330/1 from the UK Engineering and Physical Science Research Council (EPSRC) to Imperial College.

\section{REFERENCES}

[1] S. Dobson and et al., "A survey of autonomic communications," $A C M$ Transactions on Autonomous and Adaptive Systems (TAAS), vol. 1, no. 2, pp. 223-259, 2006.

[2] E. Gelenbe, "Steps towards self-aware networks," Communications of the ACM, vol. 52, pp. 66-75, July 2009.

[3] E. Gelenbe, "Sensible decisions based on qos," Computational Management Science, vol. 1, no. 1, pp. 1-14, 2003.

[4] C. Pettey, "Gartner estimates ict industry accounts for 2 percent of global co2 emissions," https://www. gartner. com/newsroom/id/503867, vol. 14, p. 2013, 2007.

[5] E. Uysal-Biyikoglu, B. Prabhakar, and A. El Gamal, "Energy-efficient packet transmission over a wireless link," IEEE/ACM Transactions on Networking (TON), vol. 10, no. 4, pp. 487-499, 2002.

[6] V. Rodoplu and T. H. Meng, "Bits-per-joule capacity of energy-limited wireless networks," Wireless Communications, IEEE Transactions on, vol. 6, no. 3, pp. 857-865, 2007.

[7] F. Meshkati, H. V. Poor, S. C. Schwartz, and N. B. Mandayam, "An energy-efficient approach to power control and receiver design in wireless data networks," Communications, IEEE Transactions on, vol. 53, no. 11, pp. 1885-1894, 2005.

[8] E. Gelenbe, K. Hussain, and V. Kaptan, "Simulating autonomous agents in augmented reality," Journal of Systems and Software, vol. 74, pp. 255268, February 2005.

[9] C. Alippi and C. Galperti, "An adaptive system for optimal solar energy harvesting in wireless sensor network nodes," Circuits and Systems I: Regular Papers, IEEE Transactions on, vol. 55, no. 6, pp. 1742-1750, 2008.

[10] W. K. Seah, Z. A. Eu, and H.-P. Tan, "Wireless sensor networks powered by ambient energy harvesting (wsn-heap)-survey and challenges," in Wireless Communication, Vehicular Technology, Information Theory and Aerospace \& Electronic Systems Technology, 2009. Wireless VITAE 2009. 1st International Conference on, pp. 1-5, IEEE, 2009.

[11] E. Gelenbe, D. Gesbert, D. Gündüz, H. Külah, and E. Uysal-Biyikoglu, "Energy harvesting communication networks, optimization and demonstration: The e-crops project," in 24th TIWDC, Tyrrhenian International Workshop 2013 on Digital Communications: Green ICT, IEEE Xplore, 2013.

[12] E. Gelenbe, "A sensor node with energy harvesting," SIGMETRICS Performance Evaluation Review, vol. 42, no. 2, pp. 37-39, 2014.

[13] E. Gelenbe, "Energy packet networks: Ict based energy allocation and storage," Green Communications and Networking, p. 186, 2012.

[14] E. Gelenbe, "The first decade of g-networks," European Journal of Operational Research, vol. 126, no. 2, pp. 231-232, 2000. 